\title{
Reducing Subspaces of Some Multiplication Operators on the Bergman Space over Polydisk
}

\author{
Yanyue Shi and Na Zhou \\ School of Mathematical Sciences, Ocean University of China, Qingdao 266000, China \\ Correspondence should be addressed to Yanyue Shi; shiyanyue@gmail.com
}

Received 27 November 2014; Revised 14 February 2015; Accepted 9 March 2015

Academic Editor: Sung G. Kim

Copyright (c) 2015 Y. Shi and N. Zhou. This is an open access article distributed under the Creative Commons Attribution License, which permits unrestricted use, distribution, and reproduction in any medium, provided the original work is properly cited.

\begin{abstract}
We consider the reducing subspaces of $M_{z^{N}}$ on $A_{\alpha}^{2}\left(\mathbb{D}^{k}\right)$, where $k \geq 3, z^{N}=z_{1}^{N_{1}} \cdots z_{k}^{N_{k}}$, and $N_{i} \neq N_{j}$ for $i \neq j$. We prove that each reducing subspace of $M_{z^{N}}$ is a direct sum of some minimal reducing subspaces. We also characterize the minimal reducing subspaces in the cases that $\alpha=0$ and $\alpha \in(-1,+\infty) \backslash \mathbb{Q}$, respectively. Finally, we give a complete description of minimal reducing subspaces of $M_{z^{N}}$ on $A_{\alpha}^{2}\left(\mathbb{D}^{3}\right)$ with $\alpha>-1$.
\end{abstract}

\section{Introduction}

Denote by $\mathbb{D}$ the open unit disk in the complex plane and $\mathrm{d} A$ the normalized area measure on $\mathbb{D}$. For $-1<\alpha<\infty$, denote $\mathrm{d} A_{\alpha}(z)=(\alpha+1)\left(1-|z|^{2}\right)^{\alpha} \mathrm{d} A(z)$. For a positive integer $k$, the weighted Bergman space $A_{\alpha}^{2}\left(\mathbb{D}^{k}\right)$ is the space of all holomorphic functions on $\mathbb{D}^{k}$ which are square integrable with respect to the measure $\mathrm{d} v_{\alpha}(z)=\mathrm{d} A_{\alpha}\left(z_{1}\right) \cdots \mathrm{d} A_{\alpha}\left(z_{k}\right)$. $A_{\alpha}^{2}\left(\mathbb{D}^{k}, \mathrm{~d} v_{\alpha}\right)$ is the Hilbert space with inner product

$$
\langle f, g\rangle_{\alpha}=\int_{\mathbb{D}^{k}} f(z) \overline{g(z)} \mathrm{d} v_{\alpha}
$$

and $\|f\|_{\alpha}^{2}=\langle f, f\rangle_{\alpha}$. In particular, if $k=1$, then $A_{\alpha}^{2}(\mathbb{D})$ is the weighted Bergman space on $\mathbb{D}$. Denote by $\mathbb{N}_{0}$ the set of all the nonnegative integers. For a $k$-dimension multi-index $\beta=\left(\beta_{1}, \ldots, \beta_{k}\right) \in \mathbb{N}_{0}^{k}\left(\beta \geq 0\right.$ means that $\beta_{i} \geq 0$ for any $i=1,2, \ldots, k)$, write $z^{\beta}=z_{1}^{\beta_{1}} \cdots z_{k}^{\beta_{k}}$ and $\gamma_{\beta}=\left\|z^{\beta}\right\|_{\alpha}^{2}$. Then $\gamma_{\beta}=\omega_{\beta_{1}} \cdots \omega_{\beta_{k}}$, where $\omega_{\beta_{i}}=\left\|z_{i}^{\beta_{i}}\right\|_{\alpha}^{2}=\beta_{i} ! \Gamma(2+\alpha) / \Gamma\left(2+\alpha+\beta_{i}\right)$. Obviously, $\left\{z^{\beta} / \sqrt{\gamma_{\beta}}\right\}_{\beta \in \mathbb{N}_{0}^{k}}$ is an orthogonal basis of $A_{\alpha}^{2}\left(\mathbb{D}^{k}\right)$.

For every bounded analytic function $\varphi$ on $\mathbb{D}^{k}$, the multiplication operator $M_{\varphi}$ is defined by

$$
M_{\varphi}(h)=\varphi h, \quad \forall h \in A_{\alpha}^{2}\left(\mathbb{D}^{k}\right) .
$$

Recall that, in a Hilbert space $\mathscr{H}$, a (closed) subspace $\mathscr{M}$ is called reducing subspace of an operator $T$ if $T(\mathscr{M}) \subset \mathscr{M}$ and $T^{*}(\mathscr{M}) \subset \mathscr{M}$. Moreover, $\mathscr{M}$ is called minimal if $\mathscr{M}$ does not contain any proper reducing subspaces other than $\{0\}$.

Although the definition of multiplication operator $M_{\varphi}$ seems simple, the invariant subspace lattice $\operatorname{Lat} M_{\varphi}$ is very complicated. Even on the Bergman space $A^{2}(\mathbb{D})$, the characterization of invariant subspaces for the Bergman shift $M_{z}$ remains a very fascinating open problem in operator theory. To get some deeper information about Lat $M_{z}$, much effort has been devoted to studying the structure of the reducing subspaces of $M_{\varphi}$ on $A^{2}(\mathbb{D})$ (see [1] and its references).

Firstly, it is proved that the multiplication operator $M_{B}$, where $B$ is the product of two Blaschke factors, has exactly two nontrivial reducing subspaces by Sun and Wang [2] and Zhu [3] independently. On the weighted sequence space $H_{\omega}^{2}$, Stessin and Zhu [4] gave a complete description of the reducing subspaces of weighted unilateral shift operators. In particular, they show that $M_{z^{n}}$ has $n$ distinct minimal reducing subspaces on $A^{2}(\mathbb{D})$. For finite Blaschke product $B, \mathrm{Hu}$ et al. [5] obtained that $M_{B}$ has at least a reducing subspace on which the restriction of $M_{B}$ is unitary equivalent to $M_{z}$. Later on, $\mathrm{Xu}$ and $\mathrm{Yan}$ [6] generalized this result to the weighted Bergman space $A_{\alpha}^{2}(\mathbb{D})$ with $\alpha \in \mathbb{N}_{0}$. In 2009, Guo et al. [7] proved that if $B$ is a Blaschke product of degree 3 , then the number of minimal reducing subspaces of $M_{B}$ is at most 3. For finite Blaschke product $B$, they also 
raised a conjecture that the number of nontrivial minimal reducing subspaces of $M_{B}$ equals the number of connected components of the Riemann surface of $B^{-1} \circ B$ over $\mathbb{D}$. By different techniques, some partial results are obtained in [810]. Finally, an affirmative answer to the conjecture is given by Douglas et al. [11]. Furthermore, when $B$ is an infinite Blaschke product, some relative results are obtained by Guo and Huang in $[12,13]$.

On $A_{\alpha}^{2}\left(\mathbb{D}^{2}\right)$, known results about the reducing subspaces of $M_{\varphi}$ are quite few. If $\varphi$ is a monomial, the reducing subspaces of $M_{\varphi}$ are characterized in [14-17]. If $\varphi=z_{1}^{m}+z_{2}^{n}$, Dan and Huang [18] described the minimal reducing subspaces of $M_{\varphi}$ and the commutant algebra $\left\{M_{\varphi}, M_{\varphi}^{*}\right\}^{\prime}$.

Let $\mathscr{M}$ be a nonzero reducing subspace of $T_{z_{1}^{m} z_{2}^{n}}$ on $A_{\alpha}^{2}\left(\mathbb{D}^{2}\right)$ with $\alpha \neq 0$. Suppose $f \in \mathscr{M}$ satisfies $\left\langle f, z_{1}^{p} z_{2}^{q}\right\rangle \neq 0$. By [16], we know that every $z_{1}^{p} z_{2}^{q}$ must be in $\mathscr{M}$. However, on the unweighted Bergman space $A^{2}\left(\mathbb{D}^{2}\right)$, it is not true. For example, $\mathscr{M}=\overline{\operatorname{span}}\left\{\left(z_{1} z_{2}^{5}+z_{1}^{3} z_{2}^{2}\right) z_{1}^{2 h} z_{2}^{3 h}: h=0,1, \ldots\right\}$ is a reducing subspace of $M_{z_{1}^{2} z_{2}^{3}}$. But $z_{1} z_{2}^{5}$ does not belong to $\mathscr{M}$.

To know more about how $\alpha$ influences the structure of reducing subspaces, we consider the reducing subspaces of $M_{z^{N}}$ over $\mathbb{D}^{k}$ for $k \geq 3$.

Fix integer $k \geq 3$ and distinct positive integers $N_{i}$ for $i=$ $1, \ldots, k$. Denote $M_{z^{N}}=M_{z_{1}^{N_{1}}} z_{2}^{N_{2}} \ldots z_{n}^{N_{k}}$ for $N=\left(N_{1}, \ldots, N_{k}\right)$. In Section 2, we prove that each reducing subspace of $M_{z^{N}}$ is a direct sum of some minimal reducing subspaces. To classify the minimal reducing subspaces, we consider three cases: (i) $\alpha$ is irrational; (ii) $\alpha=0$; (iii) $\alpha$ is rational and $\alpha \neq 0$. For cases (i) and (ii), we describe the minimal reducing subspaces of $M_{z^{N}}$. For case (iii), we find that the minimal reducing subspaces of $M_{z^{N}}$ are varied. In Section 3, we give a complete characterization of the reducing subspaces of $M_{z^{N}}$ when the dimension $k=3$.

\section{Reducing Subspaces on $A_{\alpha}^{2}\left(\mathbb{D}^{k}\right)$}

The aim of this section is to give a complete description of the reducing subspaces of $M_{z^{N}}$ on $A_{\alpha}^{2}\left(\mathbb{D}^{k}\right)$. Denote

$$
\Omega=\left\{n=\left(n_{1}, \ldots, n_{k}\right) \in \mathbb{N}_{0}^{k}: 0 \leq n_{i}<N_{i} \text { for some } i\right\} .
$$

Define an equivalence on $\Omega$ by

$$
q \sim n \Longleftrightarrow \gamma_{q+h N}=\gamma_{n+h N}, \quad \forall h \in \mathbb{N}_{0} .
$$

Write $\gamma_{n+h N}=\prod_{i=1}^{k} \omega_{n_{i}+h N_{i}}=\prod_{i=1}^{k}\left(\left(n_{i}+h N_{i}\right) ! \Gamma(2+\alpha) / \Gamma(2+\right.$ $\left.\left.\alpha+n_{i}+h N_{i}\right)\right)$ for $h \in \mathbb{N}_{0}$. For $n \in \Omega$, let

$$
\widetilde{\Im}_{n}:=\{q \in \Omega: q \sim n\}, \quad \mathscr{H}_{n}:=\overline{\operatorname{span}}\left\{z^{J}: J \in \widetilde{\Im}_{n}\right\} .
$$

Clearly, $\bigcup_{n \in F} \widetilde{\mho}_{n}=\Omega$ and $\bigoplus_{n \in F} \mathscr{H}_{n}=\overline{\operatorname{span}}\left\{z^{J}: J \in \Omega\right\}$, where $F$ is the partition of $\Omega$ by the equivalence $\sim$. Let $P_{n}$ be the orthogonal projection from $A_{\alpha}^{2}\left(\mathbb{D}^{k}\right)$ onto $\mathscr{H}_{n}$.

Theorem 1. Let $\mathscr{M}$ be a nonzero reducing subspace of $M_{z^{N}}$ on $A_{\alpha}^{2}\left(\mathbb{D}^{k}\right)$. Then, $M$ contains a minimal reducing subspace

$$
[f]=\overline{\operatorname{span}}\left\{f z^{h N}: h \in \mathbb{N}_{0}\right\}
$$

where $n \in \Omega$ and $f=\sum_{J \in \mathfrak{\Im}_{n}} b_{J} z^{J}$ with coefficients $b_{J} \in \mathbb{C}$.
Proof. Let $P_{\mathscr{M}}$ be the orthogonal projection from $A_{\alpha}^{2}\left(\mathbb{D}^{k}\right)$ onto $\mathscr{M}$. For abbreviation, we denote $M=M_{z^{N}}=$ $M_{z_{1}^{N_{1}}} z_{2}^{N_{2} \ldots z_{k}^{N_{k}}}$.

Firstly, we show that $P_{\mathscr{M}}\left(z^{m}\right) \in \mathscr{H}_{n}$ for every $m \in \mathfrak{\Im}_{n}$. Let $l=\left(l_{1}, \ldots, l_{k}\right) \in \mathbb{N}_{0}^{k}$. We only need to prove that if $\left\langle P_{\mathscr{M}} z^{m}, z^{l}\right\rangle \neq 0$, then $l \in \mathfrak{\Im}_{n}$. If $l \notin \Omega$, then $l \geq N$; that is, $l_{i} \geq N_{i}$ for $1 \leq i \leq k$. Therefore,

$$
\left\langle P_{\mathscr{M}} z^{m}, z^{l}\right\rangle=\left\langle P_{\mathscr{M}} z^{m}, M z^{l-N}\right\rangle=\left\langle P_{\mathscr{M}} M^{*} z^{m}, z^{l-N}\right\rangle=0 .
$$

If $l \in \Omega$, we find that

$$
M^{* h} M^{h}\left(z^{J}\right)=\prod_{i=1}^{k} \frac{\omega_{j_{i}+h N_{i}}}{\omega_{j_{i}}} z^{j_{i}}=\frac{\gamma_{J+h N}}{\gamma_{J}} z^{J}
$$

for $J=\left(j_{1}, \ldots, j_{k}\right) \in \mathbb{N}_{0}^{k}$. Then,

$$
\begin{aligned}
\frac{\gamma_{m+h N}}{\gamma_{m}}\left\langle P_{\mathscr{M}} z^{m}, z^{l}\right\rangle & =\left\langle P_{\mathscr{M}} M^{* h} M^{h} z^{m}, z^{l}\right\rangle \\
& =\left\langle M^{* h} M^{h} P_{\mathscr{M}} z^{m}, z^{l}\right\rangle \\
& =\left\langle P_{\mathscr{M}} z^{m}, M^{* h} M^{h} z^{l}\right\rangle \\
& =\frac{\gamma_{l+h N}}{\gamma_{l}}\left\langle P_{\mathscr{M}} z^{m}, z^{l}\right\rangle .
\end{aligned}
$$

Thus, we get that if $\left\langle P_{\mathscr{M}} z^{m}, z^{l}\right\rangle \neq 0$, then

$$
\prod_{i=1}^{k} \frac{\omega_{m_{i}+h N_{i}}}{\omega_{m_{i}}}=\prod_{i=1}^{k} \frac{\omega_{l_{i}+h N_{i}}}{\omega_{l_{i}}}, \quad \forall h \in \mathbb{N}_{0} .
$$

Since $\lim _{h \rightarrow+\infty}\left(\omega_{m_{i}+h N_{i}} / \omega_{l_{i}+h N_{i}}\right)=1$, we have $\prod_{i=1}^{k}\left(\omega_{l_{i}} / \omega_{m_{i}}\right)=$ 1. Therefore,

$$
\prod_{i=1}^{k} \omega_{l_{i}+h N_{i}}=\prod_{i=1}^{k} \omega_{m_{i}+h N_{i}}, \quad \forall h \in \mathbb{N}_{0},
$$

which implies $l \in \mathfrak{\Im}_{m}=\mathfrak{\Im}_{n}$.

Thus $P_{\mathscr{M}}\left(z^{m}\right) \in \mathscr{H}_{n}$. We also obtain that $P_{\mathscr{M}}\left(z^{l}\right) \perp \mathscr{H}_{n}$ for any $l \notin \widetilde{\mho}_{n}$.

Next, we claim that there is a nonzero function $f_{0}$ in $\mathscr{H}_{n_{0}} \cap$ $\mathscr{M}$ for some positive integer $n_{0}$.

Choose a nonzero function $f$ in $\mathscr{M}$. Let $h_{0}$ be the minimal integer such that $P_{\Omega} M^{* h_{0}}(f) \neq 0$, where $P_{\Omega}$ is the orthogonal projection from $A_{\alpha}^{2}\left(\mathbb{D}^{k}\right)$ onto $\overline{\operatorname{span}}\left\{z^{J}: J \in \Omega\right\}$. Namely, there exists $n_{0} \in \Omega$ such that $f_{0}=P_{n_{0}} M^{* h_{0}} f=P_{n_{0}} P_{\Omega} M^{* h_{0}} f=$ $\sum_{J \in \mathfrak{I}_{n_{0}}} b_{J} z^{J} \neq 0$. Then, we can prove that $f_{0}=P_{\mathscr{M}} f_{0} \in \mathscr{M}$. In fact,

(i) if $m \in \mathfrak{\Im}_{n_{0}}$, then

$$
\begin{aligned}
\left\langle P_{\mathscr{M}} f_{0}, z^{m}\right\rangle & =\left\langle P_{\mathscr{M}} P_{n_{0}} M^{* h_{0}} f, z^{m}\right\rangle \\
& =\left\langle M^{* h_{0}} f, P_{\mathscr{M}^{\prime}} P_{n_{0}} z^{m}\right\rangle \\
& =\left\langle P_{n_{0}} P_{\mathscr{M}} M^{* h_{0}} f, z^{m}\right\rangle \\
& =\left\langle f_{0}, z^{m}\right\rangle,
\end{aligned}
$$


where the second equality comes from $z^{m}, P_{\mathscr{M}}\left(z^{m}\right) \in$ $\mathscr{H}_{n}$ and the last equality comes from $M^{* h_{0}} f \in \mathscr{M}$;

(ii) if $m$ is out of $\mathfrak{\Im}_{n_{0}}$, then $\left\langle P_{\mathscr{M}} f_{0}, z^{m}\right\rangle=0=\left\langle f_{0}, z^{m}\right\rangle$.

Therefore, we get $\left[f_{0}\right] \subset \mathscr{M}$, where $\left[f_{0}\right]$ is the reducing subspace of $M$ induced by $f_{0}$. Notice that

(a) $M^{q}\left(f_{0} z^{h N}\right)=f_{0} z^{(h+q) N}$ for $h, q \geq 0$;

(b) $M^{* q}\left(f_{0} z^{h N}\right)= \begin{cases}\frac{\gamma_{n_{0}+h N}}{\gamma_{n_{0}+(h-q) N}} f_{0} z^{(h-q) N}, & \text { if } h \geq q \geq 1 \\ 0, & \text { if } 0 \leq h<q \text {; }\end{cases}$

(c) $f_{0} z^{h_{1} N} \perp f_{0} z^{h_{2} N} \quad$ with $h_{1} \neq h_{2}$, since

$$
\begin{aligned}
\left\langle f_{0} z^{h_{1} N}, f_{0} z^{h_{2} N}\right\rangle & \\
= & \left\langle M^{h_{1}} f_{0}, M^{h_{2}} f_{0}\right\rangle \\
= & \begin{cases}\frac{\gamma_{n_{0}+h_{2} N}}{\gamma_{n_{0}}}\left\langle f_{0} z^{\left(h_{1}-h_{2}\right) N}, f_{0}\right\rangle, & \text { if } h_{1}>h_{2} \geq 0 \\
\frac{\gamma_{n_{0}+h_{1} N}}{\gamma_{n_{0}}}\left\langle f_{0}, f_{0} z^{\left(h_{2}-h_{1}\right) N}\right\rangle, & \text { if } h_{2}>h_{1} \geq 0 .\end{cases}
\end{aligned}
$$

Hence, we conclude that $\left[f_{0}\right]=\overline{\operatorname{span}}\left\{f_{0} z^{h N}: h \in \mathbb{N}_{0}\right\}=$ $\bigoplus_{h=0}^{+\infty} \operatorname{span}\left\{f_{0} z^{h N}\right\} \subset \mathscr{M}$ is a minimal reducing subspace of $M$.

In the following, we will prove that each nonzero reducing subspace of $M_{z^{N}}$ is the orthogonal sum of some minimal reducing subspaces.

Theorem 2. Let $M$ be a nonzero reducing subspace of $M_{z^{N}}$ on $A_{\alpha}^{2}\left(\mathbb{D}^{k}\right)$. Then,

$$
\mathscr{M}=\bigoplus_{n \in F}\left[P_{n} \mathscr{M}\right]
$$

where $\left[P_{n} \mathscr{M}\right]$ is the reducing subspace of $M_{z^{N}}$ induced by $P_{n} \mathscr{M}$ If $P_{n} \mathscr{M} \neq\{0\}$, then

$$
\left[P_{n} \mathscr{M}\right]=\bigoplus_{h=0}^{+\infty} z^{h N} P_{n} \mathscr{M}=\bigoplus_{j=1}^{q}\left[e_{n, j}\right]
$$

where $\left\{e_{n, j}\right\}_{j=1}^{q}$ is the orthogonal basis of $P_{n} \mathscr{M}$ and $1 \leq q \leq+\infty$.

Proof. Denote $M=M_{z^{N}}$. Firstly, we know that $\left[P_{n} \mathscr{M}\right]=$ $\bigoplus_{h=0}^{+\infty} z^{h N} P_{n} \mathscr{M}$, since

(i) $z^{h_{1} N} P_{n} \mathscr{M} \perp z^{h_{2} N} P_{n} \mathscr{M}$;

(ii) $M\left(z^{h N} P_{n} \mathscr{M}\right)=z^{(h+1) N} P_{n} \mathscr{U}$;

(iii) $M^{*}\left(P_{n} \mathscr{M}\right)=\{0\}, M^{*}\left(z^{h N} P_{n} \mathscr{M}\right)=z^{(h-1) N} P_{n} \mathscr{M}$ for $h \geq 1$;

(iv) $M^{*} M\left(z^{h N} P_{n} \mathscr{M}\right)=z^{h N} P_{n} \mathscr{M}, M M^{*}\left(z^{h N} P_{n} \mathscr{M}\right)=$ $z^{h N} P_{n} \mathscr{M}$ for $h \geq 1$.
Secondly, we prove that $\mathscr{M}=\bigoplus_{n \in F}\left[P_{n} \mathscr{M}\right]$. On the one hand, in the proof of Theorem 1 , we get $P_{n} \mathscr{M} \subset \mathscr{M}$. Then, $\left[P_{n} \mathscr{M}\right] \subset \mathscr{M}$. On the other hand, if $\mathscr{M} \neq \bigoplus_{n \in F}\left[P_{n} \mathscr{M}\right]$, choose a nonzero function $f \perp \bigoplus_{n \in F}\left[P_{n} \mathscr{M}\right]$ in $\mathscr{M}$. Theorem 1 shows that there are $n_{0}$ and $h_{0}$ such that $0 \neq f_{0}=P_{n_{0}} M^{* h_{0}} f \in \mathscr{M}$. However, $\left\langle f_{0}, g\right\rangle=\left\langle f, M^{h_{0}} P_{n_{0}} g\right\rangle=0$ for $g \in \mathscr{M}$, which is a contradiction.

Finally, we prove that if $P_{n} \mathscr{M} \neq\{0\}$, then $\left[P_{n} \mathscr{M}\right]=$ $\bigoplus_{j=1}^{q}\left[e_{n, j}\right]$. Choose an orthogonal basis $\left\{e_{n, j}\right\}_{j=1}^{q}(q \leq$ $+\infty)$ of the subspace $P_{n} \mathscr{M}$. Theorem 1 shows that $\left[e_{n, j}\right]=$ $\overline{\operatorname{span}}\left\{e_{n, j} z^{h N}: h \in \mathbb{N}_{0}\right\} \subset \mathscr{M}$. We have that $\left[e_{n, p_{1}}\right] \perp\left[e_{m, p_{2}}\right]$ for $n \neq m$, since

$$
\begin{aligned}
\left\langle e_{n, p_{1}} z^{h_{1} N}, e_{m, p_{2}} z^{h_{2} N}\right\rangle & \\
= & \left\langle M^{h_{1}} e_{n, p_{1}}, M^{h_{2}} e_{m, p_{2}}\right\rangle \\
= & \begin{cases}\frac{\gamma_{m+h_{2} N}}{\gamma_{m}}\left\langle e_{n, p_{1}} z^{\left(h_{1}-h_{2}\right) N}, e_{m, p_{2}}\right\rangle, & \text { if } h_{1} \geq h_{2} \geq 0 \\
\frac{\gamma_{n+h_{1} N}}{\gamma_{n}}\left\langle e_{n, p_{1}}, e_{m, p_{2}} z^{\left(h_{2}-h_{1}\right) N}\right\rangle, & \text { if } h_{2}>h_{1} \geq 0 .\end{cases}
\end{aligned}
$$

Let $\mathscr{M}_{n}=\bigoplus_{j=1}^{q}\left[e_{n, j}\right]$. Clearly, $\mathscr{M}_{n} \subset\left[P_{n} \mathscr{M}\right]$. Assume that $\mathscr{M}_{n} \neq\left[P_{n} \mathscr{M}\right]$. Take a nonzero function $g \in\left[P_{n} \mathscr{M}\right] \ominus \mathscr{M}_{n}$. As in Theorem 1 , there is an integer $h_{0}$ such that $g_{0}:=P_{n} M^{* h_{0}} g \in$ $P_{n} \mathscr{M}$ and $g_{0} \neq 0$. Since $g \perp \mathscr{M}_{n}$, we have $\left\langle g_{0}, e_{n, j}\right\rangle=$ $\left\langle g, M^{h_{0}} P_{n} e_{n, j}\right\rangle=0$, which is in contradiction with $g_{0} \neq 0$. So we finish the proof.

From this theorem, we know that the reducing subspaces of $M_{z^{N}}$ are determined by the sets $\left\{\mathfrak{J}_{n}\right\}_{n \in \Omega}$. There arises the following question: what are the elements in the set $\mathfrak{J}_{n}$ exactly? We begin the research with the case that $\alpha$ is irrational.

Lemma 3. If $\alpha$ is irrational, then $\mathfrak{\Im}_{n}=\{n\}$ for every $n \in \Omega$.

Proof. Suppose $m \in \mathfrak{\Im}_{n}$; that is, $\gamma_{n+h N}=\gamma_{m+h N}$, for all $h \in \mathbb{N}_{0}$. Then, we have

$$
\frac{\gamma_{n+h N}}{\gamma_{n+(h+1) N}}=\frac{\gamma_{m+h N}}{\gamma_{m+(h+1) N}}, \quad \forall h \in \mathbb{N}_{0}
$$

This is equivalent to

$$
\begin{aligned}
& \prod_{i=1}^{k} \prod_{j=1}^{N_{i}} \frac{n_{i}+h N_{i}+\alpha+1+j}{n_{i}+h N_{i}+j} \\
& \quad=\prod_{i=1}^{k} \prod_{j=1}^{N_{i}} \frac{m_{i}+h N_{i}+\alpha+1+j}{m_{i}+h N_{i}+j}, \quad \forall h \in \mathbb{N}_{0} .
\end{aligned}
$$


Write

$$
\begin{aligned}
g(\lambda)= & \prod_{i=1}^{k} \prod_{j=1}^{N_{i}}\left[\left(n_{i}+\lambda N_{i}+\alpha+1+j\right)\left(m_{i}+\lambda N_{i}+j\right)\right] \\
& -\prod_{i=1}^{k} \prod_{j=1}^{N_{i}}\left[\left(m_{i}+\lambda N_{i}+\alpha+1+j\right)\left(n_{i}+\lambda N_{i}+j\right)\right] .
\end{aligned}
$$

Clearly, $g$ is a polynomial over $\mathbb{C}$ and $g(h)=0$ for any $h \in \mathbb{N}_{0}$. Fundamental theorem of algebra shows that $g(\lambda) \equiv 0$, for all $\lambda \in \mathbb{C}$. Denote

$$
\begin{aligned}
& E_{1}=\left\{\frac{n_{i}+\alpha+1+j}{N_{i}}: j=1, \ldots, N_{i}, i=1, \ldots, k\right\}, \\
& E_{2}=\left\{\frac{n_{i}+j}{N_{i}}: j=1, \ldots, N_{i}, i=1, \ldots, k\right\}, \\
& F_{1}=\left\{\frac{m_{i}+\alpha+1+j}{N_{i}}: j=1, \ldots, N_{i}, i=1, \ldots, k\right\}, \\
& F_{2}=\left\{\frac{m_{i}+j}{N_{i}}: j=1, \ldots, N_{i}, i=1, \ldots, k\right\} .
\end{aligned}
$$

Since $\alpha$ is irrational, $E_{1} \cap E_{2}=F_{1} \cap F_{2}=\emptyset$. Then, $g(\lambda) \equiv 0$ implies $E_{1} \cup F_{2}=E_{2} \cup F_{1}$. So we get $E_{1}=F_{1}$ and $E_{2}=F_{2}$.

Without loss of generality, we may assume $\max F_{2}=$ $\left(m_{k}+N_{k}\right) / N_{k}$. Then there exist nonnegative integers $i$ and $j$ making

$$
\frac{m_{i}+\alpha+1+j}{N_{i}}=\frac{n_{k}+\alpha+2}{N_{k}} .
$$

If $i \neq k$, then $\alpha=\left(\left(n_{k}+2\right) N_{i}-\left(m_{i}+1+j\right) N_{k}\right) /\left(N_{k}-N_{i}\right) \epsilon$ $\mathbb{Q}$, which is in contradiction with the assumption. So $i=k$.

Equality (21) implies $\left(m_{k}+j\right) / N_{k}=\left(n_{k}+1\right) / N_{k}$. Then, $\max E_{2} \geq\left(n_{k}+N_{k}\right) / N_{k}=\left(m_{k}+N_{k}+j-1\right) / N_{k} \geq \max F_{2}$. Hence, we get $j=1$ and $m_{k}=n_{k}$.

Therefore,

$$
\begin{aligned}
\prod_{i=1}^{k-1} \prod_{j=1}^{N_{i}} \frac{n_{i}+h N_{i}+\alpha+1+j}{n_{i}+h N_{i}+j} & \\
& =\prod_{i=1}^{k-1} \prod_{j=1}^{N_{i}} \frac{m_{i}+h N_{i}+\alpha+1+j}{m_{i}+h N_{i}+j}, \quad \forall h \in \mathbb{N}_{0} .
\end{aligned}
$$

Let

$$
\begin{aligned}
& \widetilde{E}_{1}=\left\{\frac{n_{i}+\alpha+1+j}{N_{i}}: j=1, \ldots, N_{i}, i=1, \ldots, k-1\right\}, \\
& \widetilde{E}_{2}=\left\{\frac{n_{i}+j}{N_{i}}: j=1, \ldots, N_{i}, i=1, \ldots, k-1\right\}, \\
& \widetilde{F}_{1}=\left\{\frac{m_{i}+\alpha+1+j}{N_{i}}: j=1, \ldots, N_{i}, i=1, \ldots, k-1\right\}, \\
& \widetilde{F}_{2}=\left\{\frac{m_{i}+j}{N_{i}}: j=1, \ldots, N_{i}, i=1, \ldots, k-1\right\} .
\end{aligned}
$$

Without loss of generality, assume $\max \widetilde{F}_{2}=\left(m_{k-1}+N_{k-1}\right) /$ $N_{k-1}$. As above, it is easy to get $m_{k-1}=n_{k-1}$. Applying this process again, we can prove that $m_{i}=n_{i}$ for $i=1, \ldots, k$.

By Theorems 1 and 2 and Lemma 3, we obtain the following theorem.

Theorem 4. If $\alpha$ is irrational, then each reducing subspace $\mathscr{M}$ of $M_{z^{N}}$ on $A_{\alpha}^{2}\left(\mathbb{D}^{k}\right)$ is a direct sum of some minimal reducing subspaces of the form

$$
\overline{\operatorname{span}}\left\{z^{n+h N}: h \in \mathbb{N}_{0}\right\},
$$

where $n \in \mathscr{A}=\left\{n \in \Omega: z^{n} \in \mathscr{M}\right\}$.

Proof. Lemma 3 shows that $\mathfrak{\Im}_{n}=\{n\}$. In light of Theorem 1, we have $\mathscr{A} \neq \emptyset$. For $n \in \mathscr{A}$, Theorem 2 implies that $P_{n} \mathscr{M}=$ $\operatorname{span}\left\{z^{n}\right\},\left[z^{n}\right]=\overline{\operatorname{span}}\left\{z^{n+h N}: h \in \mathbb{N}_{0}\right\}$. Thus, $\mathscr{M}=$ $\bigoplus_{n \in \mathscr{A}}\left[z^{n}\right]$.

Next, we consider the case that $\alpha=0$. Denote by $S_{k}$ the permutation group of the set $\{1,2, \ldots, k\}$. Let $\rho_{i j}(x)=(x+$ 1) $N_{i} / N_{j}-1$ for $x \in \mathbb{R}$.

Lemma 5. If $\alpha=0$, then

$$
\begin{gathered}
\widetilde{\Im}_{n}=\left\{\left(\rho_{1 \sigma(1)}\left(n_{\sigma(1)}\right), \rho_{2 \sigma(2)}\left(n_{\sigma(2)}\right), \ldots, \rho_{k \sigma(k)}\left(n_{\sigma(k)}\right)\right):\right. \\
\left.\sigma \in S_{k}\right\} .
\end{gathered}
$$

Proof. Suppose $m \in \mathfrak{\Im}_{n}$. By the definition of $\mathfrak{\Im}_{n}$, we have

$$
\prod_{i=1}^{k}\left(h N_{i}+m_{i}+1\right)=\prod_{i=1}^{k}\left(h N_{i}+n_{i}+1\right), \quad \forall h \in \mathbb{N}_{0} .
$$

Let $g(\lambda)=\prod_{i=1}^{k}\left(\lambda+\left(m_{i}+1\right) / N_{i}\right)-\prod_{i=1}^{k}\left(\lambda+\left(n_{i}+1\right) / N_{i}\right)$. We have $g(\lambda) \equiv 0$, since $g$ is a polynomial on $\mathbb{C}$ with infinitely many roots. Therefore,

$$
\left\{\frac{n_{i}+1}{N_{i}}: i=1, \ldots, k\right\}=\left\{\frac{m_{i}+1}{N_{i}}: i=1, \ldots, k\right\} .
$$

For each $j \in\{1,2, \ldots, k\}$, there is only one integer $i \in\{1,2$, $\ldots, k\}$ such that

$$
\frac{n_{j}+1}{N_{j}}=\frac{m_{i}+1}{N_{i}}
$$

that is, $m_{i}=\left(n_{j}+1\right) N_{i} / N_{j}-1=\rho_{i j}\left(n_{j}\right)$. Let

$$
\begin{gathered}
E=\left\{\left(\rho_{1 \sigma(1)}\left(n_{\sigma(1)}\right), \rho_{2 \sigma(2)}\left(n_{\sigma(2)}\right), \ldots, \rho_{k \sigma(k)}\left(n_{\sigma(k)}\right)\right):\right. \\
\left.\sigma \in S_{k}\right\} .
\end{gathered}
$$

Hence $m \in E$.

Conversely, for every $m \in E,\left(m_{i}+1\right) / N_{i}=\left(\rho_{i \sigma(i)}\left(n_{\sigma(i)}\right)+\right.$ 1) $/ N_{i}$. By definition of $\rho_{i j}\left(n_{j}\right)$, we have

$$
\frac{\rho_{i \sigma(i)}\left(n_{\sigma(i)}\right)+1}{N_{i}}=\frac{n_{\sigma(i)}+1}{N_{\sigma(i)}} .
$$

Therefore, equality (27) holds, implying $m \in \mathfrak{\Im}_{n}$. Therefore, $\mathfrak{\Im}_{n}=E$.

From this result, we find $\operatorname{Card}\left(\mathfrak{J}_{n}\right) \leq n !$. 
Example 6. Let

$$
\begin{aligned}
f(z)= & a_{1} z_{1} z_{3}+a_{2} z_{1}^{2} z_{2} z_{3}^{3}+a_{3} z_{1}^{2} z_{2}^{3} z_{3}+a_{4} z_{1} z_{2} z_{3}^{5} \\
& +a_{5} z_{1} z_{3}^{11}+a_{6} z_{1}^{5} z_{3}^{3}+a_{7} z_{1}^{11} z_{3} .
\end{aligned}
$$

Denote by $\mathscr{M}=[f]$ the reducing subspace of $M_{z_{1}^{3} z_{2} z_{3}^{6}}$ on $A^{2}\left(\mathbb{D}^{3}\right)$ induced by $f$. Let

(i) $\mathscr{M}_{1}=\overline{\operatorname{span}}\left\{f_{1}(z) z_{1}^{3 h} z_{2}^{h} z_{3}^{6 h}: h=0,1,2, \ldots\right\}$ for $f_{1}(z)=z_{1} z_{3}$

(ii) $\mathscr{M}_{2}=\overline{\operatorname{span}}\left\{f_{2}(z) z_{1}^{3 h} z_{2}^{h} z_{3}^{6 h}: h=0,1,2, \ldots\right\}$ for $f_{2}(z)=a_{2} z_{1}^{2} z_{2} z_{3}^{3}+a_{4} z_{1} z_{2} z_{3}^{5}+a_{5} z_{1} z_{3}^{11}+a_{6} z_{1}^{5} z_{3}^{3} ;$

(iii) $\mathscr{M}_{3}=\overline{\operatorname{span}}\left\{f_{3}(z) z_{1}^{3 h} z_{2}^{h} z_{3}^{6 h}: h=0,1,2, \ldots\right\}$ for $f_{3}(z)=a_{3} z_{1}^{2} z_{2}^{3} z_{3}+a_{7} z_{1}^{11} z_{3}$.

Then, $\mathscr{M}=\bigoplus_{i=1}^{3} \mathscr{M}_{i}$.

Proof. Let $n=\left(n_{1}, n_{2}, n_{3}\right)=(2,1,3)$ and let $m=\left(m_{1}, m_{2}, m_{3}\right)=$ $(11,0,1)$. It is easy to check that

$$
\begin{aligned}
& (1,1,5)=\left(\rho_{13}\left(n_{3}\right), \rho_{22}\left(n_{2}\right), \rho_{31}\left(n_{1}\right)\right), \\
& (5,0,3)=\left(\rho_{12}\left(n_{2}\right), \rho_{21}\left(n_{1}\right), \rho_{33}\left(n_{3}\right)\right), \\
& (1,0,11)=\left(\rho_{13}\left(n_{3}\right), \rho_{21}\left(n_{1}\right), \rho_{32}\left(n_{2}\right)\right) .
\end{aligned}
$$

That is,

$$
\mathfrak{J}_{n}=\{(2,1,3),(1,1,5),(1,0,11),(5,0,3)\} .
$$

Similarly,

$$
\widetilde{J}_{m}=\{(11,0,1),(2,3,1),(0,3,5),(0,0,23)\} .
$$

By Lemma 5, we get $f_{2}(z)=a_{2} z_{1}^{2} z_{2} z_{3}^{3}+a_{4} z_{1} z_{2} z_{3}^{5}+a_{5} z_{1} z_{3}^{11}+$ $a_{6} z_{1}^{5} z_{3}^{3} \in[f]$ and $f_{3}(z)=a_{3} z_{1}^{2} z_{2}^{3} z_{3}+a_{7} z_{1}^{11} z_{3} \in[f]$. Therefore, $a_{1} z_{1} z_{3} \in[f]$. Notice that

$$
M^{* h} M^{q} f(z)= \begin{cases}\sum_{i=1}^{3} \mu_{i} f_{i}(z) z^{(q-h) N}, & \text { if } 0 \leq h \leq q \\ 0, & \text { if } 0 \leq q<h,\end{cases}
$$

where $\mu_{1}=\gamma_{(1,0,1)+q N} / \gamma_{(1,0,1)+(q-h) N}, \mu_{2}=\gamma_{(2,3,1)+q N} /$ $\gamma_{(2,3,1)+(q-h) N}$, and $\mu_{3}=\gamma_{(11,0,1)+q N} / \gamma_{(11,0,1)+(q-h) N}$. So

$$
[f]=\bigoplus_{i=1}^{3}\left[f_{i}\right]=\bigoplus_{i=1}^{3} M_{i}
$$

If $\alpha$ is a nonzero rational number, the structure of minimal reducing subspace turns to be more complicated. In particular, we will study the reducing subspaces of $M_{z^{N}}$ on $A_{\alpha}^{2}\left(\mathbb{D}^{3}\right)$ in the next section.

\section{Reducing Subspaces on $A_{\alpha}^{2}\left(\mathbb{D}^{3}\right)$}

Let $\alpha \neq 0$ be rational. We consider the reducing subspaces of $M_{z^{N}}$ on $A_{\alpha}^{2}\left(\mathbb{D}^{3}\right)$. Recall

$$
\Omega=\left\{n=\left(n_{1}, n_{2}, n_{3}\right) \in \mathbb{N}_{0}^{3}: 0 \leq n_{i}<N_{i} \text { for some } i\right\},
$$

and $\mathfrak{\Im}_{n}=\{q \in \Omega: q \sim n\}$; that is, $m \in \mathfrak{\Im}_{n}$ if and only if $\gamma_{m+h N}=\gamma_{n+h N}$ for $h \in \mathbb{N}_{0}$. For every $n \in \Omega$, if $m \in \mathfrak{\Im}_{n}$, we assume that $m_{i} \neq n_{i}$ for $i=1,2,3$. Otherwise, if there exists $j$ such that $m_{j}=n_{j}$, we can prove that $m_{i}=n_{i}$ for $i=1,2,3$ as in [16]. Since $\gamma_{\beta}=\prod_{i=1}^{3} \omega_{\beta_{i}}=\prod_{i=1}^{3}\left(\beta_{i} ! \Gamma(2+\alpha) / \Gamma\left(2+\alpha+\beta_{i}\right)\right)$ and $\omega_{\beta_{i}}$ are decreasing as $\beta_{i}$ is increasing, there exist $i$ and $j$ satisfying $n_{i}>m_{i}$ and $n_{j}<m_{j}$.

This section is organized as follows. Firstly, we consider $m \in \mathfrak{J}_{n}$ under the assumption that $n_{1}>m_{1}, n_{2}>m_{2}$, and $m_{3}>n_{3}$. Let $Q_{1}=\{1,2, \ldots, n, \ldots\}, Q_{2}=\left\{\alpha \in \mathbb{Q} \backslash Q_{1}: \alpha>0\right\}$, and $Q_{3}=(-1,0) \cap \mathbb{Q}$. We give a description of $m \in \mathfrak{\Im}_{n}$ in the cases that $\alpha$ is in $Q_{1}, Q_{2}$, and $Q_{3}$, respectively. Secondly, we get all the possible cases by symmetry (see Corollaries 11 and 13). Finally, we obtain $\operatorname{Card}\left(\mathfrak{\Im}_{n}\right) \leq 2$ and Theorem 14 .

Lemma 7. Let $\alpha \in Q_{2}$ and let $n \in \Omega$. If $m \in \mathfrak{\Im}_{n}$ satisfies $n_{1}>m_{1}, n_{2}>m_{2}$, and $m_{3}>n_{3}$, then one of the following statements holds:

(1) $m=n-(1,1-1)$;

(2) $m=n-(1,1,-2)$.

Proof. Let $m \in \mathfrak{\Im}_{n}$. By definition of $\mathfrak{\mho}_{n}$, as in Lemma 3, we have

$$
\begin{aligned}
\prod_{i=1}^{2} \prod_{j=1}^{n_{i}-m_{i}} \frac{n_{i}+\alpha+2-j+\lambda N_{i}}{m_{i}+j+h N_{i}} \\
\quad=\prod_{j=1}^{m_{3}-n_{3}} \frac{m_{3}+\alpha+2-j+\lambda N_{3}}{n_{3}+j+h N_{3}}
\end{aligned}
$$

for any $\lambda \in \mathbb{C}$. Denote

$$
\begin{aligned}
& E_{i}=\left\{\frac{n_{i}+\alpha+1}{N_{i}}, \frac{n_{i}+\alpha}{N_{i}}, \ldots, \frac{m_{i}+\alpha+2}{N_{i}}\right\}, \\
& E_{3}=\left\{\frac{m_{3}}{N_{3}}, \frac{m_{3}-1}{N_{3}}, \ldots, \frac{n_{3}+1}{N_{3}}\right\}, \\
& F_{i}=\left\{\frac{n_{i}}{N_{i}}, \frac{n_{i}-1}{N_{i}}, \ldots, \frac{m_{i}+1}{N_{i}}\right\}, \\
& F_{3}=\left\{\frac{m_{3}+\alpha+1}{N_{3}}, \frac{m_{3}+\alpha}{N_{3}}, \ldots, \frac{n_{3}+\alpha+2}{N_{3}}\right\},
\end{aligned}
$$

for $i=1,2$. Then,

$$
\bigsqcup_{i=1}^{3} E_{i}=\bigsqcup_{i=1}^{3} F_{i},
$$

where $\bigsqcup$ denotes the disjoint union. Since $\alpha>0$ is not an integer, $E_{i} \cap F_{i}=\emptyset$ for $i=1,2,3$. 
It is easy to see that

$$
\begin{aligned}
\max & \left\{\frac{n_{1}+\alpha+1}{N_{1}}, \frac{n_{2}+\alpha+1}{N_{2}}, \frac{m_{3}}{N_{3}}\right\} \\
& =\max \left\{\frac{n_{1}}{N_{1}}, \frac{n_{2}}{N_{2}}, \frac{m_{3}+\alpha+1}{N_{3}}\right\} .
\end{aligned}
$$

Since $\left(n_{i}+\alpha+1\right) / N_{i}>n_{i} / N_{i}$ for $i=1,2$ and $\left(m_{3}+\alpha+1\right) / N_{3}>$ $m_{3} / N_{3}$, we have

$$
\frac{m_{3}+\alpha+1}{N_{3}} \in\left\{\frac{n_{1}+\alpha+1}{N_{1}}, \frac{n_{2}+\alpha+1}{N_{2}}\right\} .
$$

Without loss of generality, assume

$$
\frac{n_{1}+\alpha+1}{N_{1}}=\frac{m_{3}+\alpha+1}{N_{3}}
$$

Firstly, we prove that $n_{1}-m_{1}=1$ by contradiction. Otherwise, if $n_{1}-m_{1} \geq 2$, then

$$
\begin{aligned}
\max & \left(\bigsqcup_{i=1}^{3} E_{i} \backslash\left\{\frac{n_{1}+\alpha+1}{N_{1}}\right\}\right) \\
& =\max \left(\bigsqcup_{i=1}^{3} F_{i} \backslash\left\{\frac{m_{3}+\alpha+1}{N_{3}}\right\}\right) .
\end{aligned}
$$

Since $\max \left\{\left(n_{1}+\alpha\right) / N_{1},\left(n_{2}+\alpha+1\right) / N_{2}\right\}>\max \left\{n_{1} / N_{1}, n_{2} / N_{2}\right\}$, we have $m_{3}-n_{3} \geq 2$ and

$$
\begin{aligned}
\max & \left\{\frac{n_{1}+\alpha}{N_{1}}, \frac{n_{2}+\alpha+1}{N_{2}}, \frac{m_{3}}{N_{3}}\right\} \\
& =\max \left\{\frac{n_{1}}{N_{1}}, \frac{n_{2}}{N_{2}}, \frac{m_{3}+\alpha}{N_{3}}\right\}=\frac{m_{3}+\alpha}{N_{3}}>\frac{m_{3}}{N_{3}} .
\end{aligned}
$$

Therefore, $\left(m_{3}+\alpha\right) / N_{3} \in\left\{\left(n_{1}+\alpha\right) / N_{1},\left(n_{2}+\alpha+1\right) / N_{2}\right\}$. Since $N_{1} \neq N_{3}$, it holds that

$$
\frac{m_{3}+\alpha}{N_{3}}=\frac{n_{2}+\alpha+1}{N_{2}}
$$

We will find the contradictions under the assumptions (a) $n_{2}-m_{2} \geq 2$ and (b) $n_{2}-m_{2}=1$, respectively.

(a) If $n_{2}-m_{2} \geq 2$, then $\max \left\{\left(n_{1}+\alpha\right) / N_{1},\left(n_{2}+\alpha\right) /\right.$ $\left.N_{2}, m_{3} / N_{3}\right\}>\max \left\{n_{1} / N_{1}, n_{2} / N_{2}\right\}$. So $m_{3}-n_{3} \geq 3$ and

$$
\begin{aligned}
\max & \left\{\frac{n_{1}+\alpha}{N_{1}}, \frac{n_{2}+\alpha}{N_{2}}, \frac{m_{3}}{N_{3}}\right\} \\
& =\max \left\{\frac{n_{1}}{N_{1}}, \frac{n_{2}}{N_{2}}, \frac{m_{3}+\alpha-1}{N_{3}}\right\} .
\end{aligned}
$$

Since $N_{2} \neq N_{3}$, we have

$$
\frac{m_{3}+\alpha-1}{N_{3}}=\frac{n_{1}+\alpha}{N_{1}}
$$

By (43) and (48), we get $1 / N_{1}=2 / N_{3}$ and $m_{3} / N_{3}>n_{1} /$ $N_{1}$. It follows that

$$
\begin{aligned}
\max & \left\{\frac{n_{1}+\alpha-1}{N_{1}}, \frac{n_{2}+\alpha}{N_{2}}, \frac{m_{3}}{N_{3}}\right\} \\
& \geq \max \left\{\frac{n_{2}+\alpha}{N_{2}}, \frac{m_{3}}{N_{3}}\right\}>\max \left\{\frac{n_{1}}{N_{1}}, \frac{n_{2}}{N_{2}}\right\} .
\end{aligned}
$$

Thus, $m_{3}-n_{3} \geq 4$ and

$$
\frac{m_{3}+\alpha-2}{N_{3}}=\max \left\{\frac{n_{1}}{N_{1}}, \frac{n_{2}}{N_{2}}, \frac{m_{3}+\alpha-2}{N_{3}}\right\} .
$$

By $N_{1} \neq N_{3}$ and equality (48), we conclude that

$$
\frac{m_{3}+\alpha-2}{N_{3}}=\frac{n_{2}+\alpha}{N_{2}} .
$$

Equalities (46) and (51) imply $2 / N_{3}=1 / N_{2}$. Thus, $N_{2}=$ $N_{1}$, which is in contradiction with the assumption.

(b) Suppose $n_{2}-m_{2}=1$. Notice that $E_{2}=\left\{\left(n_{2}+\alpha+1\right) /\right.$ $\left.N_{2}\right\} \subset F_{3}$.

If $F_{2}=\left\{n_{2} / N_{2}\right\} \subset E_{1}$, then equality (40) implies $F_{1}=E_{3}$. Equivalently, $n_{1} / N_{1}=m_{3} / N_{3}$ and $\left(n_{1}-1\right) / N_{1}=\left(m_{3}-1\right) / N_{3}$. Hence, $N_{1}=N_{3}$, which is impossible.

If $F_{2}=\left\{n_{2} / N_{2}\right\} \subset E_{3}$, then $F_{1} \cup F_{2}=E_{3}$. It follows that $\max \left\{n_{1} / N_{1}, n_{2} / N_{2}\right\}=m_{3} / N_{3}$. Since $N_{1} \neq N_{3}$, equality (43) implies $m_{3} / N_{3}=n_{2} / N_{2}$. Therefore, $n_{1} / N_{1}=\left(m_{3}-1\right) / N_{3}$ and $\left(n_{1}-1\right) / N_{1}=\left(m_{3}-2\right) / N_{3}$. Then, $N_{3}=N_{1}$, which is a contradiction.

Summing up, we must have $n_{1}-m_{1}=1$.

Next, we prove that $n_{2}-m_{2}=1$.

If $F_{1} \subset E_{2}$, then $F_{2}=E_{3}$; that is,

$$
\frac{n_{2}}{N_{2}}=\frac{m_{3}}{N_{3}}
$$

In this case, $n_{2}-m_{2}=m_{3}-n_{3}=1$. Otherwise, $n_{2} / N_{2}=m_{3} / N_{3}$ and $\left(n_{2}-1\right) / N_{2}=\left(m_{3}-1\right) / N_{3}$, which is in contradiction with $N_{2} \neq N_{3}$. that

If $F_{1} \subset E_{3}$, then $E_{2}=F_{3} \backslash\left\{\left(m_{3}+\alpha+1\right) / N_{3}\right\}$. It follows

$$
\frac{n_{2}+\alpha+1}{N_{2}}=\frac{m_{3}+\alpha}{N_{3}} \text {. }
$$

In this case, $n_{2}-m_{2}=1$ and $m_{3}-n_{3}=2$. Or else, $\left(n_{2}+\alpha+\right.$ $1) / N_{2}=\left(m_{3}+\alpha\right) / N_{3}$ and $\left(n_{2}+\alpha\right) / N_{2}=\left(m_{3}+\alpha-1\right) / N_{3}$, which is in contradiction with $N_{2} \neq N_{3}$. So we get the desired results.

Lemma 8. Fix $\alpha \in Q_{1}$ and $n \in \Omega$. If $m \in \mathfrak{\Im}_{n}$ satisfies $n_{1}>m_{1}$, $n_{2}>m_{2}$, and $m_{3}>n_{3}$, then one of the following statements holds:

(1) $m=n-(1,1-1)$;

(2) $m=n-(1,1,-2)$. 
Proof. Let $k_{i}=\min \left\{\alpha+1,\left|n_{i}-m_{i}\right|\right\}$ for $i=1,2,3$. Then,

$$
\begin{aligned}
\prod_{i=1}^{2} \prod_{j=1}^{k_{i}} \frac{n_{i}+\alpha+2-j+\lambda N_{i}}{m_{i}+j+\lambda N_{i}} \\
\quad=\prod_{j=1}^{k_{3}} \frac{m_{3}+\alpha+2-j+\lambda N_{3}}{n_{3}+j+\lambda N_{3}}
\end{aligned}
$$

for $\lambda \in \mathbb{C}$. Let

$$
\begin{aligned}
& \widetilde{E}_{i}=\left\{\frac{n_{i}+\alpha+1}{N_{i}}, \frac{n_{i}+\alpha}{N_{i}}, \ldots, \frac{n_{i}+\alpha+2-k_{i}}{N_{i}}\right\} ; \\
& \widetilde{E}_{3}=\left\{\frac{n_{3}+1}{N_{3}}, \frac{n_{3}+2}{N_{3}}, \ldots, \frac{n_{3}+k_{3}}{N_{3}}\right\} ; \\
& \widetilde{F}_{i}=\left\{\frac{m_{i}+1}{N_{i}}, \frac{m_{i}+2}{N_{i}}, \ldots, \frac{m_{i}+k_{i}}{N_{i}}\right\} ; \\
& \widetilde{F}_{3}=\left\{\frac{m_{3}+\alpha+1}{N_{3}}, \frac{m_{3}+\alpha}{N_{3}}, \ldots, \frac{m_{3}+\alpha+2-k_{3}}{N_{3}}\right\}
\end{aligned}
$$

for $i=1,2$. Then,

$$
\bigsqcup_{i=1}^{3} \widetilde{E}_{i}=\bigsqcup_{i=1}^{3} \widetilde{F}_{i}
$$

and $\widetilde{E}_{i} \cap \widetilde{F}_{i}=\emptyset$ for $i=1,2,3$. As in Lemma 7, we assume equality (43) holds. Then, we can prove that $\left(k_{1}, k_{2}, k_{3}\right)=$ $(1,1,2)$ or $\left(k_{1}, k_{2}, k_{3}\right)=(1,1,1)$.

Since $\alpha+1>1$, we have $k_{1}=n_{1}-m_{1}=1$ and $k_{2}=$ $n_{2}-m_{2}=1$. It means $\operatorname{Card}\left(\widetilde{E}_{i}\right)=\operatorname{Card}\left(\widetilde{F}_{i}\right)=1$ for $i=1,2$.

If $k_{3}=m_{3}-n_{3}$, then statement (1) or statement (2) holds.

If $k_{3}=\alpha+1$, then $\operatorname{Card}\left(\widetilde{E}_{3}\right)=\operatorname{Card}\left(\widetilde{F}_{3}\right)=k_{3}=2$ and $\alpha=1$. Equalities (43) and (53) imply that

$$
\begin{aligned}
& \frac{n_{1}+2}{N_{1}}=\frac{m_{3}+2}{N_{3}}, \\
& \frac{n_{2}+2}{N_{2}}=\frac{m_{3}+1}{N_{3}} .
\end{aligned}
$$

Since $N_{1}, N_{2}$, and $N_{3}$ are distinct, equality (56) shows that

$$
\begin{aligned}
& \frac{n_{2}}{N_{2}}=\frac{n_{3}+2}{N_{3}}, \\
& \frac{n_{1}}{N_{1}}=\frac{n_{3}+1}{N_{3}} .
\end{aligned}
$$

Then, we have $N_{1}: N_{2}: N_{3}=2: 6: 3$ and $\left(n_{1}, n_{2}, m_{3}, n_{3}\right)=$ $r\left(N_{1}, N_{2}, N_{3}, N_{3}\right)+(0,2,1,-1)$. In this case, $m_{3}-n_{3}=2=k_{3}$. Hence, statement (2) holds.

Lemma 9. Fix a rational number $\alpha>0$ and a vector $n \in \Omega$. If $m=n-(1,1,-1) \in \mathfrak{J}_{n}$, then one of the following statements holds:

(1) $1 / N_{1}+1 / N_{2}-1 / N_{3}=0$ and

$$
n \in\left\{r\left(N_{1}, N_{2}, N_{3}\right)+\left((\alpha+2) \frac{N_{1}}{N_{3}}-\alpha-1, \frac{N_{2}}{N_{3}}, 0\right): r \in \mathbb{R}\right\} \text {, }
$$

(2) $1 / N_{1}+1 / N_{2}-1 / N_{3}=0$ and

$n \in\left\{r\left(N_{1}, N_{2}, N_{3}\right)+\left(\frac{N_{1}}{N_{3}},(\alpha+2) \frac{N_{2}}{N_{3}}-\alpha-1,0\right): r \in \mathbb{R}\right\}$.

Proof. As in Lemma 7, we have $\left(m_{3}+\alpha+1\right) / N_{3} \in\left\{\left(n_{1}+\alpha+\right.\right.$ 1) $\left./ N_{1},\left(n_{2}+\alpha+1\right) / N_{2}\right\}$.

If $\left(n_{1}+\alpha+1\right) / N_{1}=\left(m_{3}+\alpha+1\right) / N_{3}$, Lemmas 7 and 8 show that

$$
\begin{gathered}
\frac{n_{1}+\alpha+1}{N_{1}}=\frac{m_{3}+\alpha+1}{N_{3}}, \\
\frac{m_{3}}{N_{3}}=\frac{n_{2}}{N_{2}}, \\
\frac{n_{1}}{N_{1}}=\frac{n_{2}+\alpha+1}{N_{2}} .
\end{gathered}
$$

Therefore,

$$
\begin{aligned}
\left(n_{1}, n_{2}, m_{3}\right)= & r\left(N_{1}, N_{2}, N_{3}\right) \\
& +\left((\alpha+2) \frac{N_{1}}{N_{3}}-\alpha-1, \frac{N_{2}}{N_{3}}, 1\right),
\end{aligned}
$$

where $r \in \mathbb{R}$ and $1 / N_{1}+1 / N_{2}-1 / N_{3}=0$. Statement (1) holds. If $\left(n_{2}+\alpha+1\right) / N_{2}=\left(m_{3}+\alpha+1\right) / N_{3}$, then we have

$$
\begin{gathered}
\frac{n_{2}+\alpha+1}{N_{2}}=\frac{m_{3}+\alpha+1}{N_{3}}, \\
\frac{m_{3}}{N_{3}}=\frac{n_{1}}{N_{1}}, \\
\frac{n_{2}}{N_{2}}=\frac{n_{1}+\alpha+1}{N_{1}} .
\end{gathered}
$$

In this case,

$$
\begin{aligned}
\left(n_{1}, n_{2}, m_{3}\right)= & r\left(N_{1}, N_{2}, N_{3}\right) \\
& +\left(\frac{N_{1}}{N_{3}},(\alpha+2) \frac{N_{2}}{N_{3}}-\alpha-1,1\right),
\end{aligned}
$$

where $r \in \mathbb{R}$ and $1 / N_{1}+1 / N_{2}-1 / N_{3}=0$. So statement (2) holds.

Lemma 10. Fix a rational number $\alpha>0$ and a vector $n \in \Omega$. If $m=n-(1,1,-2) \in \mathfrak{\Im}_{n}$, then one of the following statements holds:

(1) $N_{1}: N_{2}: N_{3}=\alpha(\alpha+1):(\alpha+1)(\alpha+2): \alpha(\alpha+2)$ and

$$
n \in\left\{r\left(N_{1}, N_{2}, N_{3}\right)+\left(0, \frac{N_{2}}{N_{3}},-1\right): r \in \mathbb{R}\right\},
$$


(2) $N_{1}: N_{2}: N_{3}=(\alpha+1)(\alpha+2): \alpha(\alpha+1): \alpha(\alpha+2)$ and

$$
n \in\left\{r\left(N_{1}, N_{2}, N_{3}\right)+\left(\frac{N_{1}}{N_{3}}, 0,-1\right): r \in \mathbb{R}\right\}
$$

In this case, $1 / N_{1}+1 / N_{2}-2 / N_{3}=0$.

Proof. As in Lemma 8, if $\left(n_{1}+\alpha+1\right) / N_{1}=\left(m_{3}+\alpha+1\right) / N_{3}$, then $\left(n_{2}+\alpha+1\right) / N_{2}=\left(m_{3}+\alpha\right) / N_{3}$. Since $N_{1} \neq N_{3}$, we have $n_{1} / N_{1} \neq m_{3} / N_{3}$. Similarly, equality (53) implies that $n_{2} / N_{2} \neq$ $\left(m_{3}-1\right) / N_{3}$. Therefore,

$$
\begin{gathered}
\frac{n_{1}+\alpha+1}{N_{1}}=\frac{m_{3}+\alpha+1}{N_{3}}, \\
\frac{n_{2}+\alpha+1}{N_{2}}=\frac{m_{3}+\alpha}{N_{3}}, \\
\frac{n_{2}}{N_{2}}=\frac{m_{3}}{N_{3}}, \\
\frac{n_{1}}{N_{1}}=\frac{m_{3}-1}{N_{3}} .
\end{gathered}
$$

Hence,

$$
\left(n_{1}, n_{2}, m_{3}\right)=r\left(N_{1}, N_{2}, N_{3}\right)+\left(0, \frac{N_{2}}{N_{3}}, 1\right),
$$

where $r \in \mathbb{R}$ and $N_{1}: N_{2}: N_{3}=\alpha(\alpha+1):(\alpha+1)(\alpha+2): \alpha(\alpha+$ $2)$. In this case, $1 / N_{1}+1 / N_{2}-2 / N_{3}=0$. Thus, we get (1).

If $\left(n_{2}+\alpha+1\right) / N_{2}=\left(m_{3}+\alpha+1\right) / N_{3}$, then it is easy to check that (2) holds.

By symmetry, we can get the following corollary.

Corollary 11. Fix a rational number $\alpha>0$ and $n \in \Omega$. If $m \in \mathfrak{J}_{n} \backslash\{n\}$, then one of the following statements holds.

(1) $m_{\sigma(1)}=n_{\sigma(1)}-1, m_{\sigma(2)}=n_{\sigma(2)}-1, m_{\sigma(3)}=n_{\sigma(3)}+1$, where $\sigma \in S_{k}$. In this case, $1 / N_{\sigma(1)}+1 / N_{\sigma(2)}-1 / N_{\sigma(3)}=$ 0 and

$$
\begin{aligned}
& n \in\left\{r\left(N_{1}, N_{2}, N_{3}\right)+\left(k_{1}, k_{2}, k_{3}\right):\right. \\
& \left\{\begin{array}{ll}
k_{\sigma(1)}=\frac{(\alpha+2) N_{\sigma(1)}}{N_{\sigma(3)}}-\alpha-1 & \\
k_{\sigma(2)}=\frac{N_{\sigma(2)}}{N_{\sigma(3)}} & r \in \mathbb{R} \\
k_{\sigma(3)}=0, &
\end{array}\right\} .
\end{aligned}
$$

(2) $m_{\sigma(1)}=n_{\sigma(1)}+1, m_{\sigma(2)}=n_{\sigma(2)}+1, m_{\sigma(3)}=n_{\sigma(3)}-1$, where $\sigma \in S_{k}$. In this case, $1 / N_{\sigma(1)}+1 / N_{\sigma(2)}-1 / N_{\sigma(3)}=$ 0 and

$$
\begin{aligned}
& n \in\left\{r\left(N_{1}, N_{2}, N_{3}\right)+\left(k_{1}, k_{2}, k_{3}\right):\right. \\
& \left\{\begin{array}{ll}
k_{\sigma(1)}=\frac{(\alpha+2) N_{\sigma(1)}}{N_{\sigma(3)}}-\alpha-2 & \\
k_{\sigma(2)}=\frac{N_{\sigma(2)}}{N_{\sigma(3)}}-1 & r \in \mathbb{R} \\
k_{\sigma(3)}=1, &
\end{array}\right\} .
\end{aligned}
$$

(3) $m_{\sigma(1)}=n_{\sigma(1)}-1, m_{\sigma(2)}=n_{\sigma(2)}-1, m_{\sigma(3)}=n_{\sigma(3)}+2$, where $\sigma \in S_{k}$. In this case, $N_{\sigma(1)}: N_{\sigma(2)}: N_{\sigma(3)}=\alpha(\alpha+$ 1) : $(\alpha+1)(\alpha+2): \alpha(\alpha+2)$ and

$$
n \in\left\{r\left(N_{1}, N_{2}, N_{3}\right)+\left(k_{1}, k_{2}, k_{3}\right):\right.
$$

$$
\left\{\begin{array}{l}
k_{\sigma(1)}=0 \\
k_{\sigma(2)}=\frac{N_{\sigma(2)}}{N_{\sigma(3)}} \\
k_{\sigma(3)}=-1,
\end{array} \quad r \in \mathbb{R}\right\} .
$$

(4) $m_{\sigma(1)}=n_{\sigma(1)}+1, m_{\sigma(2)}=n_{\sigma(2)}+1, m_{\sigma(3)}=n_{\sigma(3)}-2$, where $\sigma \in S_{k}$. In this case, $N_{\sigma(1)}: N_{\sigma(2)}: N_{\sigma(3)}=\alpha(\alpha+$ 1) : $(\alpha+1)(\alpha+2): \alpha(\alpha+2)$ and

$$
\begin{aligned}
& n \in\left\{r\left(N_{1}, N_{2}, N_{3}\right)+\left(k_{1}, k_{2}, k_{3}\right):\right. \\
& \left\{\begin{array}{l}
k_{\sigma(1)}=-1 \\
k_{\sigma(2)}=\frac{N_{\sigma(2)}}{N_{\sigma(3)}}-1 \quad r \in \mathbb{R} \\
k_{\sigma(3)}=1,
\end{array}\right\} .
\end{aligned}
$$

Lemma 12. Fix a rational number $\alpha \in(-1,0)$ and $n \in \Omega$. If there exists $m \in \mathfrak{\Im}_{n}$ such that $n_{1}>m_{1}, n_{2}>m_{2}$, and $m_{3}>n_{3}$, then one of the following statements holds:

(1) $m=n-(1,1,-1)$. In this case, $1 / N_{1}+1 / N_{2}+1 / N_{3}=0$ and

$$
\begin{aligned}
n \in\left\{r\left(N_{1}, N_{2}, N_{3}\right)\right. \\
\left.\quad+\left((\alpha+2) \frac{N_{1}}{N_{3}}-\alpha-1, \frac{N_{2}}{N_{3}}, 0\right): r \in \mathbb{R}\right\},
\end{aligned}
$$




$$
\begin{aligned}
& \text { or } \\
& n \in\left\{r\left(N_{1}, N_{2}, N_{3}\right)\right. \\
& \left.+\left(\frac{N_{1}}{N_{3}},(\alpha+2) \frac{N_{2}}{N_{3}}-\alpha-1,0\right): r \in \mathbb{R}\right\},
\end{aligned}
$$

(2) $m=n-(2,1,-1)$. In this case, $N_{1}: N_{2}: N_{3}=-\alpha(\alpha+$ $2):(\alpha+1)(\alpha+2):-\alpha(\alpha+1)$ and

$$
n \in\left\{r\left(N_{1}, N_{2}, N_{3}\right)+\left(1,(\alpha+1) \frac{N_{2}}{N_{1}},-1\right): r \in \mathbb{R}\right\} .
$$

(3) $m=n-(1,2,-1)$. In this case, $N_{1}: N_{2}: N_{3}=(\alpha+$ 1) $(\alpha+2):-\alpha(\alpha+2):-\alpha(\alpha+1)$ and

$$
n \in\left\{r\left(N_{1}, N_{2}, N_{3}\right)+\left((\alpha+1) \frac{N_{1}}{N_{2}}, 1,-1\right): r \in \mathbb{R}\right\} .
$$

Proof. Define $E_{i}$ and $F_{i}$ as in the proof of Lemma 7. Then, $\bigsqcup_{i=1}^{3} E_{i}=\bigsqcup_{i=1}^{3} F_{i}$ and $E_{i} \cap F_{i}=\emptyset$ for $i=1,2,3$. Assume that

$$
\frac{n_{1}+\alpha+1}{N_{1}}=\frac{m_{3}+\alpha+1}{N_{3}} \in F_{3} .
$$

Then,

$$
\begin{aligned}
\max & \left(\bigsqcup_{i=1}^{3} E_{i} \backslash\left\{\frac{n_{1}+\alpha+1}{N_{1}}\right\}\right) \\
& =\max \left(\bigsqcup_{i=1}^{3} F_{i} \backslash\left\{\frac{m_{3}+\alpha+1}{N_{3}}\right\}\right) .
\end{aligned}
$$

Since $\left(n_{2}+\alpha+1\right) / N_{2}>n_{2} / N_{2},\left(n_{1}+\alpha\right) / N_{1}<n_{1} / N_{1}$, and $\left(m_{3}+\alpha\right) / N_{3}<m_{3} / N_{3}$, we have

$$
\max \left\{\frac{n_{2}+\alpha+1}{N_{2}}, \frac{m_{3}}{N_{3}}\right\}=\frac{n_{1}}{N_{1}} .
$$

By (77) and $N_{1} \neq N_{3}$, we get

$$
\frac{n_{1}}{N_{1}}=\frac{n_{2}+\alpha+1}{N_{2}} \in E_{2} \text {. }
$$

(a) If $n_{1}-m_{1}=1$, then $F_{2}=E_{3}$. Therefore, $n_{2}-m_{2}=$ $m_{3}-n_{3}=1$. Otherwise, $n_{2} / N_{2}=m_{3} / N_{3}$ and $\left(n_{2}-1\right) / N_{2}=$ $\left(m_{3}-1\right) / N_{3}$, which is in contradiction with $N_{2} \neq N_{3}$.

In this case, we have

$$
\begin{gathered}
\frac{n_{1}+\alpha+1}{N_{1}}=\frac{m_{3}+\alpha+1}{N_{3}}, \\
\frac{n_{1}}{N_{1}}=\frac{n_{2}+\alpha+1}{N_{2}}, \\
\frac{n_{2}}{N_{2}}=\frac{m_{3}}{N_{3}} .
\end{gathered}
$$

Hence,

$$
\left(n_{1}, n_{2}, m_{3}\right)=r\left(N_{1}, N_{2}, N_{3}\right)+\left((\alpha+2) \frac{N_{1}}{N_{3}}-\alpha-1, \frac{N_{2}}{N_{3}}, 1\right),
$$

where $r \in \mathbb{R}$ and

$$
\frac{1}{N_{1}}+\frac{1}{N_{2}}=\frac{1}{N_{3}}
$$

or, equivalently,

$$
\begin{gathered}
n=r\left(N_{1}, N_{2}, N_{3}\right)+\left((\alpha+2) \frac{N_{1}}{N_{3}}-\alpha-1, \frac{N_{2}}{N_{3}}, 0\right) ; \\
m=\left(n_{1}-1, n_{2}-1, n_{3}+1\right) .
\end{gathered}
$$

So (i) holds.

(b) Assume $n_{1}-m_{1} \geq 2$.

Since $\left(n_{1}-1\right) / N_{1}<\left(n_{1}+\alpha\right) / N_{1},\left(n_{2}+\alpha\right) / N_{2}<n_{2} / N_{2}$, and $\left(m_{3}+\alpha\right) / N_{3}<m_{3} / N_{3}$, we get

$$
\begin{aligned}
\max & \left\{\frac{n_{1}+\alpha}{N_{1}}, \frac{n_{2}+\alpha}{N_{2}}, \frac{m_{3}}{N_{3}}\right\} \\
& =\max \left\{\frac{n_{1}+\alpha}{N_{1}}, \frac{m_{3}}{N_{3}}\right\}=\frac{n_{2}}{N_{2}} \\
& =\max \left\{\frac{n_{1}-1}{N_{1}}, \frac{n_{2}}{N_{2}}, \frac{m_{3}+\alpha}{N_{3}}\right\} .
\end{aligned}
$$

We prove that $\left(n_{1}+\alpha\right) / N_{1}=n_{2} / N_{2}$ by contradiction. Otherwise, if $m_{3} / N_{3}=n_{2} / N_{2}$, then equalities (77) and (80) imply equality (83). Therefore, $1 / N_{3}>1 / N_{1}$ and $\left(n_{1}+\right.$ $\alpha) / N_{1}>\left(m_{3}+\alpha\right) / N_{3}$. Since $-1<\alpha<0$, we have

$$
\begin{gathered}
\max \left\{\frac{n_{1}+\alpha}{N_{1}}, \frac{m_{3}-1}{N_{3}}\right\}>\max \left\{\frac{n_{1}-1}{N_{1}}, \frac{m_{3}+\alpha}{N_{3}}\right\}, \\
\max \left\{\frac{n_{1}+\alpha}{N_{1}}, \frac{n_{2}+\alpha}{N_{2}}, \frac{m_{3}-1}{N_{3}}\right\} \\
>\max \left\{\frac{n_{1}-1}{N_{1}}, \frac{n_{2}-1}{N_{2}}, \frac{m_{3}+\alpha}{N_{3}}\right\},
\end{gathered}
$$

which contradicts $\bigsqcup_{i=1}^{3} E_{i}=\bigsqcup_{i=1}^{3} F_{i}$. Thus,

$$
\frac{n_{1}+\alpha}{N_{1}}=\frac{n_{2}}{N_{2}} \text {. }
$$

Combining equality (80), we get

$$
-\frac{\alpha}{N_{1}}=\frac{\alpha+1}{N_{2}} .
$$

In the following, we prove that $n_{2}-m_{2}=1$ by contradiction. Assume $n_{2}-m_{2} \geq 2$. $\operatorname{By}\left(n_{1}-1\right) / N_{1}>\left(n_{1}+\alpha-1\right) / N_{1}$, $\left(n_{2}+\alpha\right) / N_{2}>\left(n_{2}-1\right) / N_{2}$, and $m_{3} / N_{3}>\left(m_{3}+\alpha\right) / N_{3}$, we get

$$
\max \left\{\frac{n_{2}+\alpha}{N_{2}}, \frac{m_{3}}{N_{3}}\right\}=\max \left\{\frac{n_{1}-1}{N_{1}}, \frac{n_{2}-1}{N_{2}}\right\}=\frac{n_{1}-1}{N_{1}} .
$$


If $\left(n_{1}-1\right) / N_{1}=\left(n_{2}+\alpha\right) / N_{2}$, combining the equalities (87) and (88), we get $N_{1}=N_{2}$, which is a contradiction.

If $\left(n_{1}-1\right) / N_{1}=m_{3} / N_{3}$, equality (77) shows that $N_{1}: N_{3}=$ $(\alpha+2):(\alpha+1)$. We also have $m_{3}-n_{3} \geq 2$, since

$$
\max \left\{\frac{n_{1}+\alpha-1}{N_{1}}, \frac{n_{2}+\alpha}{N_{2}}\right\}>\max \left\{\frac{n_{1}-2}{N_{1}}, \frac{n_{2}-1}{N_{2}}\right\} .
$$

Therefore,

$$
\begin{aligned}
\max & \left\{\frac{n_{1}+\alpha-1}{N_{1}}, \frac{n_{2}+\alpha}{N_{2}}, \frac{m_{3}-1}{N_{3}}\right\} \\
& =\frac{m_{3}+\alpha}{N_{3}}=\max \left\{\frac{n_{1}-2}{N_{1}}, \frac{n_{2}-1}{N_{2}}, \frac{m_{3}+\alpha}{N_{3}}\right\} .
\end{aligned}
$$

We conclude that

$$
\frac{m_{3}+\alpha}{N_{3}}=\max \left\{\frac{n_{2}+\alpha}{N_{2}}, \frac{n_{1}+\alpha-1}{N_{1}}\right\} .
$$

If $\left(m_{3}+\alpha\right) / N_{3}=\left(n_{1}+\alpha-1\right) / N_{1}$, equality (77) shows that

$$
N_{1}: N_{3}=2: 1 \text {. }
$$

So $(\alpha+2):(\alpha+1)=2: 1$; that is, $\alpha=0$ which is in contradiction with $\alpha<0$.

If $\left(m_{3}+\alpha\right) / N_{3}=\left(n_{2}+\alpha\right) / N_{2}$, the fact

$$
\begin{aligned}
\max & \left\{\frac{n_{1}+\alpha-1}{N_{1}}, \frac{n_{2}+\alpha-1}{N_{2}}, \frac{m_{3}-1}{N_{3}}\right\} \\
& =\max \left\{\frac{n_{1}-2}{N_{1}}, \frac{n_{2}-1}{N_{2}}, \frac{m_{3}+\alpha-1}{N_{3}}\right\},
\end{aligned}
$$

implies that

$$
\max \left\{\frac{n_{1}+\alpha-1}{N_{1}}, \frac{m_{3}-1}{N_{3}}\right\}=\frac{n_{2}-1}{N_{2}} .
$$

However, $\left(n_{2}-1\right) / N_{2} \neq\left(m_{3}-1\right) / N_{3}$ because of $\left(m_{3}+\alpha\right) / N_{3}=$ $\left(n_{2}+\alpha\right) / N_{2}$ and $N_{2} \neq N_{3}$ and $\left(n_{2}-1\right) / N_{2} \neq\left(n_{1}+\alpha-\right.$ 1) $/ N_{1}$, because of equality (87) and $N_{1} \neq N_{2}$. We also get a contradiction. Hence, $n_{2}-m_{2}=1$.

Therefore, $E_{2}=\left\{\left(n_{2}+\alpha+1\right) / N_{2}\right\} \subset F_{1}$ and $F_{2}=\left\{n_{2} / N_{2}\right\} \subset$ $E_{1}$. Further, $F_{1} \backslash\left\{n_{1} / N_{1}\right\}=E_{3}$. It follows that $m_{3}-n_{3}=1$, $n_{1}-m_{1}=2$, and $m_{3} / N_{3}=\left(n_{1}-1\right) / N_{1}$. Summing up, we have

$$
\begin{gathered}
\frac{n_{1}+\alpha+1}{N_{1}}=\frac{m_{3}+\alpha+1}{N_{3}}, \\
\frac{n_{1}}{N_{1}}=\frac{n_{2}+\alpha+1}{N_{2}}, \\
\frac{n_{2}}{N_{2}}=\frac{n_{1}+\alpha}{N_{1}}, \\
\frac{m_{3}}{N_{3}}=\frac{n_{1}-1}{N_{1}} .
\end{gathered}
$$

Hence, $\left(n_{1}, n_{2}, m_{3}\right)=r\left(N_{1}, N_{2}, N_{3}\right)+\left(1,(\alpha+1) N_{2} / N_{1}, 0\right)$, where $r \in \mathbb{R}$ and

$$
N_{1}: N_{2}: N_{3}=-\alpha(\alpha+2):(\alpha+1)(\alpha+2):-\alpha(\alpha+1) \text {. }
$$

Thus, (2) holds. In this case, $2 / N_{1}+1 / N_{2}-1 / N_{3}=0$.
Similarly, if $\left(n_{2}+\alpha+1\right) / N_{2}=\left(m_{3}+\alpha+1\right) / N_{3}$, by symmetry, we can get (3) and another part of (1).

By symmetry, we also have the following corollary.

Corollary 13. Let $\alpha \in(-1,0) \cap \mathbb{Q}$ and let $n \in \Omega$. If there exists $m \in \mathfrak{\Im}_{n}$ such that $n \neq m$, then there is a permutation $\sigma \in S_{k}$ such that one of the following statements holds.

(1) $1 / N_{\sigma(1)}+1 / N_{\sigma(2)}-1 / N_{\sigma(3)}=0$ and

$n \in\left\{r\left(N_{1}, N_{2}, N_{3}\right)+\left(k_{1}, k_{2}, k_{3}\right):\right.$

$$
\left\{\begin{array}{ll}
k_{\sigma(1)}=\frac{(\alpha+2) N_{\sigma(1)}}{N_{\sigma(3)}}-\alpha-1 & \\
k_{\sigma(2)}=\frac{N_{\sigma(2)}}{N_{\sigma(3)}} & r \in \mathbb{R} \\
k_{\sigma(3)}=0, &
\end{array}\right\} .
$$

In this case, $m_{\sigma(1)}=n_{\sigma(1)}-1, m_{\sigma(2)}=n_{\sigma(2)}-1$, and $m_{\sigma(3)}=n_{\sigma(3)}+1$.

(2) $1 / N_{\sigma(1)}+1 / N_{\sigma(2)}-1 / N_{\sigma(3)}=0$ and

$n \in\left\{r\left(N_{1}, N_{2}, N_{3}\right)+\left(k_{1}, k_{2}, k_{3}\right):\right.$

$$
\left\{\begin{array}{ll}
k_{\sigma(1)}=\frac{(\alpha+2) N_{\sigma(1)}}{N_{\sigma(3)}}-\alpha-2 & \\
k_{\sigma(2)}=\frac{N_{\sigma(2)}}{N_{\sigma(3)}}-1 & r \in \mathbb{R} \\
k_{\sigma(3)}=1, &
\end{array}\right\} .
$$

In this case, $m_{\sigma(1)}=n_{\sigma(1)}+1, m_{\sigma(2)}=n_{\sigma(2)}+1$, and $m_{\sigma(3)}=n_{\sigma(3)}-1$.

(3) $N_{\sigma(1)}: N_{\sigma(2)}: N_{\sigma(3)}=-\alpha(\alpha+2):(\alpha+1)(\alpha+2):-\alpha(\alpha+$ 1) and

$$
\begin{aligned}
& n \in\left\{r\left(N_{1}, N_{2}, N_{3}\right)+\left(k_{1}, k_{2}, k_{3}\right):\right. \\
& \left\{\begin{array}{l}
k_{\sigma(1)}=1 \\
k_{\sigma(2)}=\frac{(\alpha+1) N_{\sigma(2)}}{N_{\sigma(1)}} \\
k_{\sigma(3)}=-1,
\end{array} \quad r \in \mathbb{R}\right\} .
\end{aligned}
$$

In this case, $m_{\sigma(1)}=n_{\sigma(1)}-2, m_{\sigma(2)}=n_{\sigma(2)}-1$, and $m_{\sigma(3)}=n_{\sigma(3)}+1$. 
(4) $N_{\sigma(1)}: N_{\sigma(2)}: N_{\sigma(3)}=-\alpha(\alpha+2):(\alpha+1)(\alpha+2):-\alpha(\alpha+$ 1) and

$$
\begin{aligned}
& n \in\left\{r\left(N_{1}, N_{2}, N_{3}\right)+\left(k_{1}, k_{2}, k_{3}\right):\right. \\
& \left\{\begin{array}{l}
k_{\sigma(1)}=-1 \\
k_{\sigma(2)}=\frac{(\alpha+1) N_{\sigma(2)}}{N_{\sigma(1)}}-1 \quad r \in \mathbb{R} \\
k_{\sigma(3)}=0,
\end{array}\right\} .
\end{aligned}
$$

In this case, $m_{\sigma(1)}=n_{\sigma(1)}+2, m_{\sigma(2)}=n_{\sigma(2)}+1$, and $m_{\sigma(3)}=n_{\sigma(3)}-1$.

By careful computation, we find that each choice of $n$ and $N$ cannot simultaneously satisfy two of the statements in Corollary 11 or Corollary 13. So $\operatorname{Card}\left(\mathfrak{\Im}_{n}\right) \leq 2$. Moreover, there are finite numbers of $n \in \Omega$ such that $\operatorname{Card}\left(\mathfrak{\Im}_{n}\right)=2$. Denote

$$
\begin{aligned}
& \text { (i) } \mathscr{M}_{n}=\overline{\operatorname{span}}\left\{z^{n+h N}: h \in \mathbb{N}_{0}\right\} \text {; } \\
& \text { (ii) } \mathscr{H}_{n}=\overline{\operatorname{span}}\left\{\left(a z^{n}+b z^{n-(i, j, k)}\right) z^{h N}: h \in \mathbb{N}_{0}\right\},(i, j, k) \text { is } \\
& \quad \text { a permutation of }\{1,1,-1\} \text { or }\{1,1,-2\} \text {; } \\
& \text { (iii) } \mathcal{N}_{n}=\overline{\operatorname{span}}\left\{\left(a z^{n}+b z^{n-(i, j, k)}\right) z^{h N}: h \in \mathbb{N}_{0}\right\},(i, j, k) \text { is } \\
& \text { a permutation of }\{1,1,-1\} \text { or }\{2,1,-1\} .
\end{aligned}
$$

Theorem 2 implies the following statements.

(a) If $\alpha>0$, then each reducing subspace of $M_{z^{N}}$ is the direct sum of some minimal reducing as in (i) and (ii), where the number of reducing subspaces as (ii) is finite.

(b) If $-1<\alpha<0$, then each reducing subspace of $M_{z^{N}}$ is the direct sum of some minimal reducing as in (i) and (iii), where the number of reducing subspaces as (iii) is finite.

Finally, we consider the reducing subspaces from the viewpoint of von Neumann algebras. Denote by $\mathscr{W}^{*}\left(z^{N}\right)$ the von Neumann algebra generated by $M_{z^{N}}$ and $v^{*}\left(z^{N}\right)$ the commutant of $\mathscr{W}^{*}\left(z^{N}\right)$. Then $v^{*}\left(z^{N}\right)$ is a von Neumann algebra, and it is generated by its self-adjoint projections. For each reducing subspace $\mathscr{M}$ of $M_{z^{N}}$, denote by $P_{\mathscr{M}}$ the orthogonal projection from $A_{\alpha}^{2}\left(\mathbb{D}^{3}\right)$ onto $\mathscr{M}$. It is known that $P_{\mathscr{M}}$ is a self-adjoint projection in $\nu^{*}\left(z^{N}\right)$. Conversely, if $P$ is a self-adjoint projection in $\nu^{*}\left(z^{N}\right)$, then the range of $P$ is a reducing subspace of $M_{z^{N}}$. So our results can be written in the following form.

Theorem 14. The von Neumann algebra $v^{*}\left(z^{N}\right)$ is $*-$ isomorphic to

$$
\bigoplus_{n=1}^{m} M_{2}(\mathbb{C}) \bigoplus\left(\bigoplus_{n=1}^{+\infty} \mathbb{C}\right)
$$

where $0 \leq m<+\infty$.

\section{Conflict of Interests}

The authors declare that there is no conflict of interests regarding the publication of this paper.

\section{Acknowledgments}

This paper is supported by the Fundamental Research Funds for the Central Universities (Grant no. 201213011), NSFC (nos. 11201438, 11171315), and Shandong Province Young Scientist Research Award Fund (BS2012SF031).

\section{References}

[1] K. Guo and H. Huang, "Commutants, reducing subspaces and von Neumann algebras: based on multiplication operators on the Bergman space," Preprint.

[2] S. L. Sun and Y. Wang, "Reducing subspaces of certain analytic Toeplitz operators on the Bergman space," Northeastern Mathematical Journal, vol. 14, no. 2, pp. 147-158, 1998.

[3] K. Zhu, "Reducing subspaces for a class of multiplication operators," Journal of the London Mathematical Society, vol. 62, no. 2, pp. 553-568, 2000.

[4] M. Stessin and K. Zhu, "Reducing subspaces of the weighted shift operators," Proceedings of the American Mathematical Society, vol. 130, no. 9, pp. 2631-2639, 2002.

[5] J. Hu, S. Sun, X. Xu, and D. Yu, "Reducing subspace of analytic Toeplitz operators on the Bergman space," Integral Equations and Operator Theory, vol. 49, no. 3, pp. 387-395, 2004.

[6] A. Xu and C. Yan, "Reducing subspace of analytic Toeplitz operators on weighted Bergman spaces," Chinese Annals of Mathematics, Series A, vol. 30, pp. 639-646, 2009.

[7] K. Guo, S. Sun, D. Zheng, and C. Zhong, "Multiplication operators on the Bergman space via the Hardy space of the bidisk," Journal für Die Reine und Angewandte Mathematik, vol. 2009, no. 628, pp. 129-168, 2009.

[8] R. G. Douglas, S. Sun, and D. Zheng, "Multiplication operators on the Bergman space via analytic continuation," Advances in Mathematics, vol. 226, no. 1, pp. 541-583, 2011.

[9] K. Guo and H. Huang, "On multiplication operators of the Bergman space: similarity, unitary equivalence and reducing subspaces," Journal of Operator Theory, vol. 65, no. 2, pp. 355378, 2011.

[10] S. Sun, D. Zheng, and C. Zhong, "Classification of reducing subspaces of a class of multiplication operators on the Bergman space via the hardy space of the bidisk," Canadian Journal of Mathematics, vol. 62, no. 2, pp. 415-438, 2010.

[11] R. G. Douglas, M. Putinar, and K. Wang, "Reducing subspaces for analytic multipliers of the Bergman space," Journal of Functional Analysis, vol. 263, no. 6, pp. 1744-1765, 2012.

[12] K. Guo and H. Huang, "Multiplication operators defined by covering maps on the Bergman space: the connection between operator theory and von Neumann algebras," Journal of Functional Analysis, vol. 260, no. 4, pp. 1219-1255, 2011.

[13] K. Guo and H. Huang, "Geometric constructions of thin Blaschke products and reducing subspace problem," Proceedings of the London Mathematical Society, vol. 109, no. 4, pp. 10501091, 2014.

[14] Y. Lu and X. Zhou, "Invariant subspaces and reducing subspaces of weighted Bergman space over bidisk," Journal of the Mathematical Society of Japan, vol. 62, no. 3, pp. 745-765, 2010. 
[15] L. Shan, "Reducing subspaces for a class of analytic Toeplitz operators on bidisc," Journal of Fudan University, vol. 42, no. 2, pp. 196-200, 2003.

[16] Y. Shi and Y. Lu, "Reducing subspaces for Toeplitz operators on the polydisk," Bulletin of the Korean Mathematical Society, vol. 50, no. 2, pp. 687-696, 2013.

[17] X. Zhou, Y. Shi, and Y. Lu, "Invariant subspaces and reducing subspaces of weighted Bergman space over polydisc," Scientia Sinica Mathematica, vol. 41, no. 5, pp. 427-438, 2011.

[18] H. Dan and H. Huang, "Multiplication operators defined by a class of polynomials on $L_{\alpha}^{2}\left(D^{2}\right)$," Integral Equations and Operator Theory, vol. 80, no. 4, pp. 581-601, 2014. 


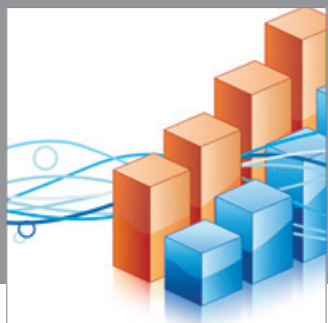

Advances in

Operations Research

mansans

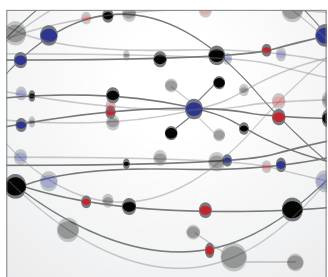

The Scientific World Journal
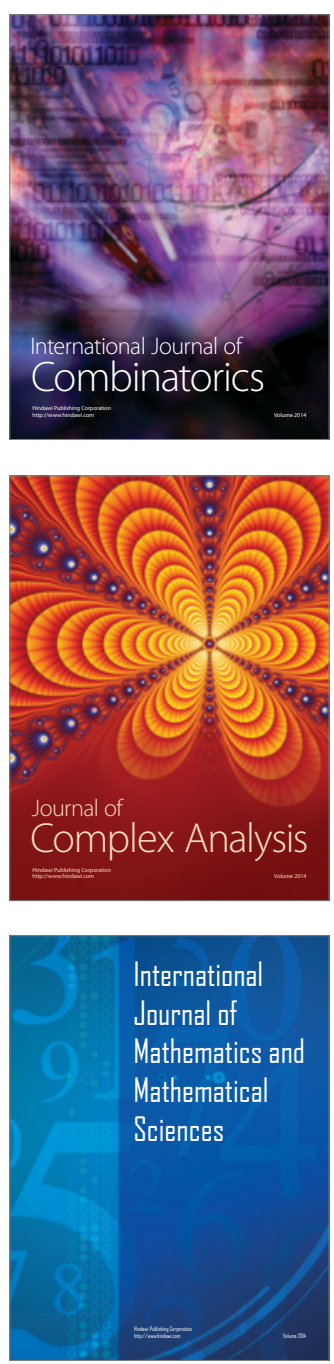
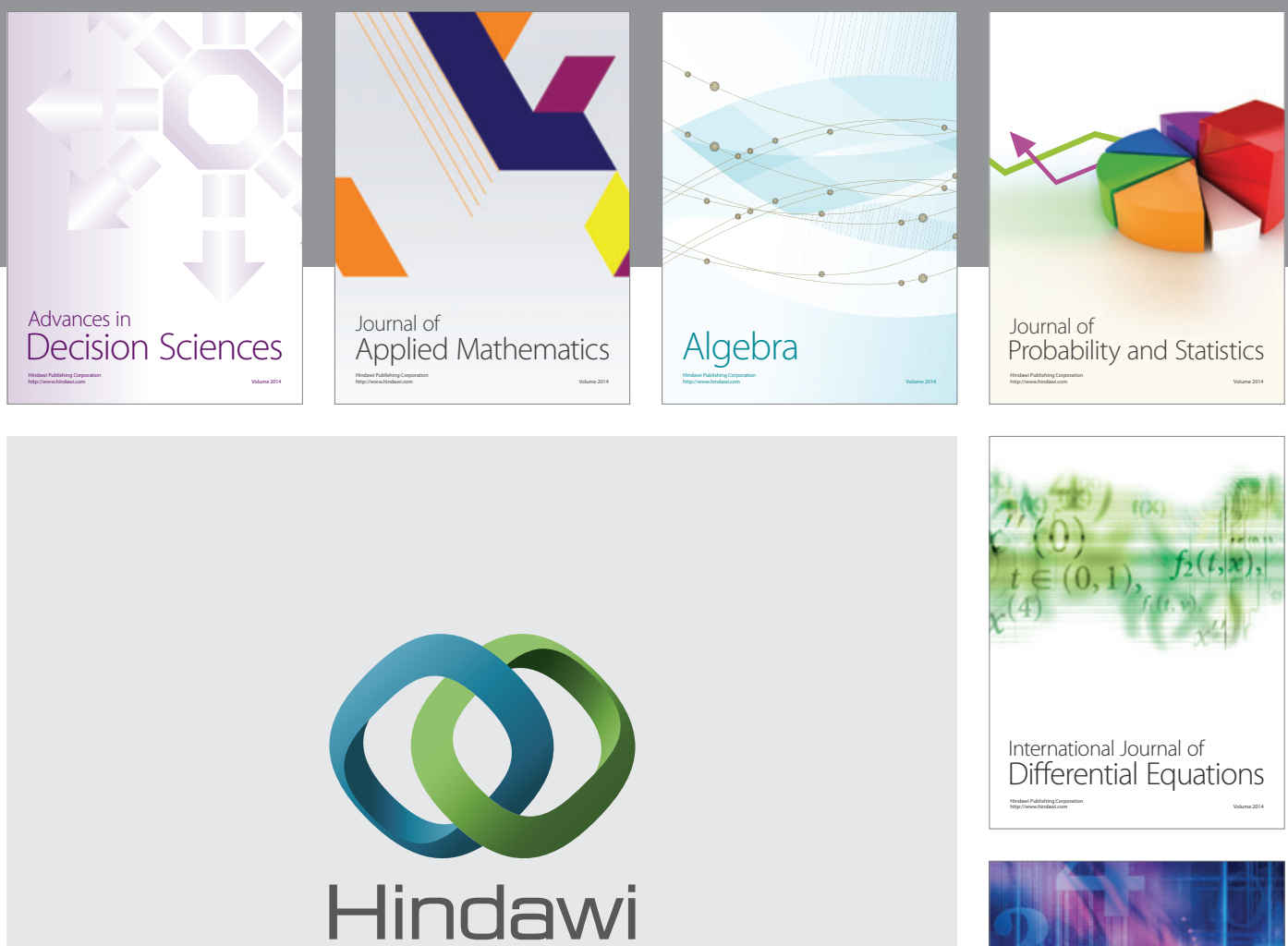

Submit your manuscripts at http://www.hindawi.com
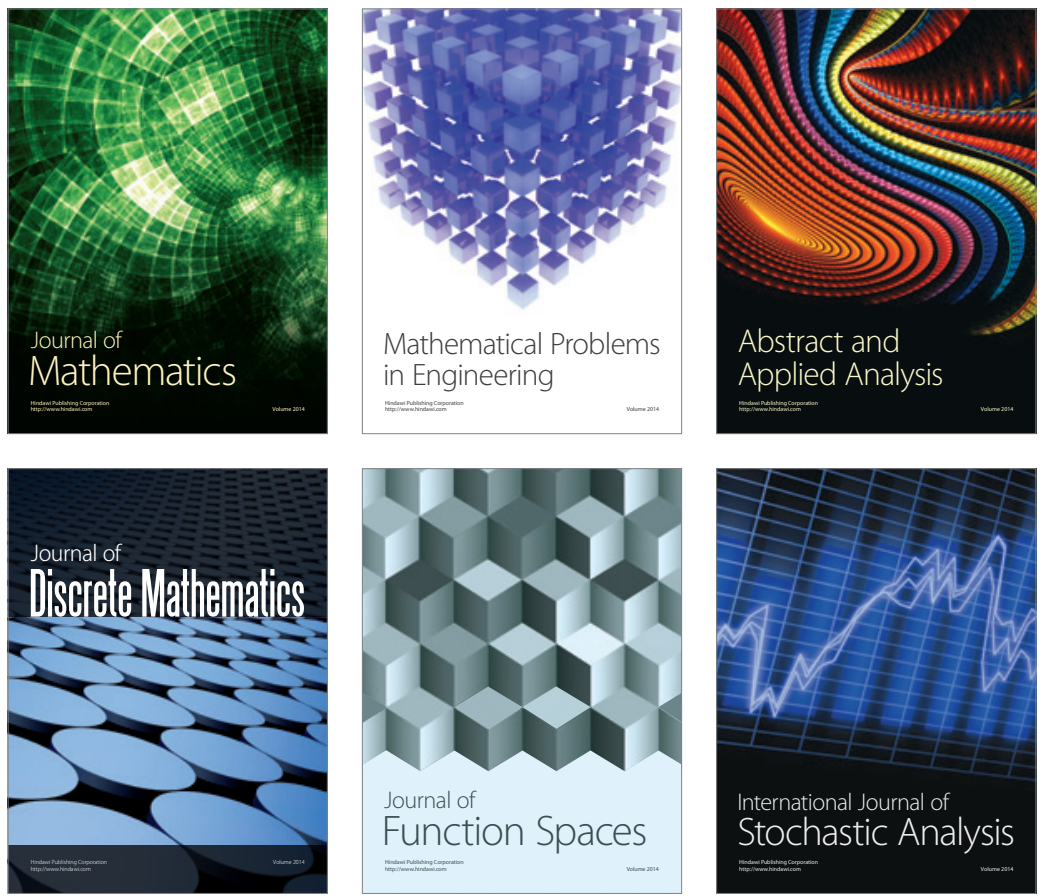

Journal of

Function Spaces

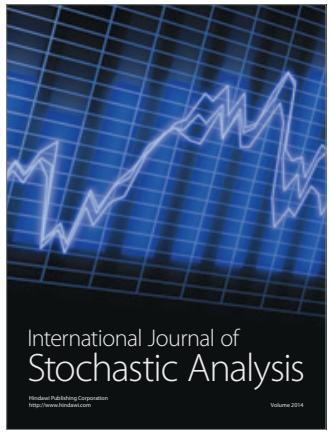

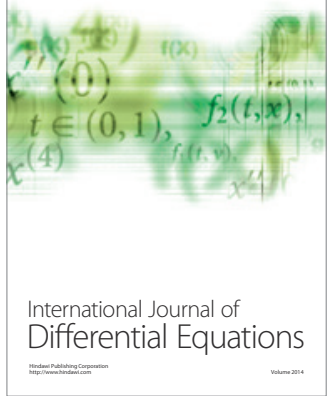
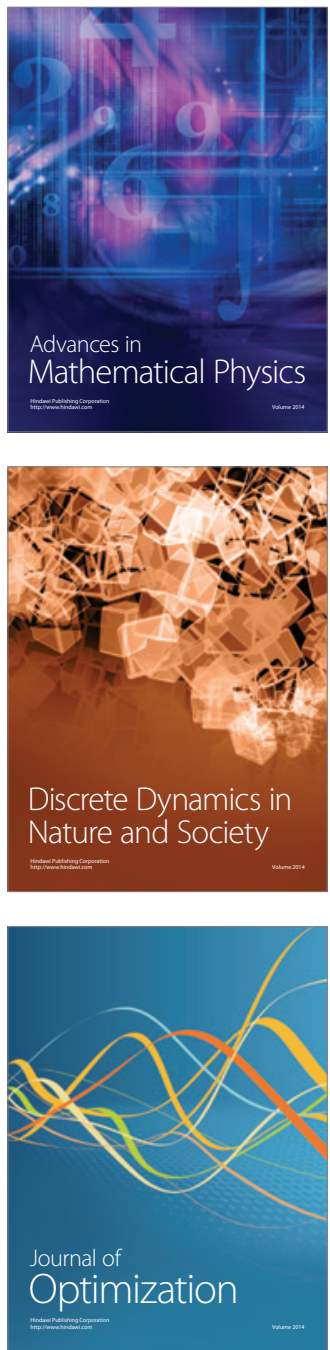